\title{
PRODUÇÃO DE COMPOSTOS ORGÂNICOS VOLÁTEIS (COV's) POR Phormidium sp. EM BIORREATORES HETEROTRÓFICOS
}

\author{
A. B. SANTOS ${ }^{1}$, A. S. FERNANDES ${ }^{1}$, M. B. FAGUNDES ${ }^{1}$, S. R. KACHUK-SILVA ${ }^{1}$, R. \\ WAGNER ${ }^{1}$, E. JACOB-LOPES ${ }^{1}$, L. Q. ZEPKA ${ }^{1}$. \\ ${ }^{1}$ Universidade Federal de Santa Maria, Departamento Tecnologia e Ciência dos Alimentos \\ E-mail para contato: lqz@pq.cnpq.br
}

\begin{abstract}
RESUMO - A elucidação da fase gasosa do sistema de cultivo heterotrófico é importante na consolidação da tecnologia para a exploração industrial. 0 objetivo do trabalho foi avaliar a produção de COV's pela microalga Phormidium sp. em cultivo heterotrófico empregando glicose como fonte de carbono. Os experimentos foram realizados em biorreator descontínuo com aeração contínua e ausência de luminosidade. Os COV's foram isolados por microextração em fase sólida (fibra DVB/Car/PDMS), analisados por cromatografia gasosa acoplada a um detector de massas (SPME-GC-MS). A amostragem foi realizada a cada 24 horas no período de 7 dias correspondendo as fases de crescimento. Foram identificados 54 compostos, como álcoois $(36,7 \%)$, cetonas $(28,6 \%)$, e aldeídos $(6,2 \%)$, sendo os picos majoritários identificados como acetaldeído, 3-hidroxi-2-butanona e 3-metil-1butanol.
\end{abstract}

\section{INTRODUÇÃO}

As microalgas são importantes recursos biológicos devido seu grande potencial para aplicações biotecnológicas e isolamento de produtos naturais bioquimicamente ativos (Borowitzka, 2013; Burja et al., 2001). O gênero Phormidium sp. é uma cianobactéria filamentosa não ramificada que pode ser encontrada em solos, rochas úmidas, lamas, plantas aquáticas e algumas espécies em ambientes extremos como os solos de desertos (Guiry \& Guiry, 2013; Thomazeu et al., 2010). Estes microrganismos são principalmente fotoautotróficos, mas um grande número deles possuem a habilidade de manter sua estrutura na ausência de luz, sendo assim capaz de crescer heterotroficamente, mantendo-se a partir da assimilação de substratos orgânicos (Wen \& Chen, 2003). A cianobactéria em questão, faz parte deste grupo com potencial de exploração do metabolismo heterotrófico.

A utilização do cultivo heterotrófico elimina requisitos de luminosidade e pode aumentar consideravelmente as taxas de crescimento de massa celular, além de permitir a utilização de qualquer fermentador como um biorreator o que possibilita uma significativa redução de custos para a maioria dos processos devido a simplicidade de operação (Morales-Sanchéz et al., 2013; Cheng et al., 2009; Perez-Garcia et al., 2011). A habilidade das microalgas em utilizar substratos orgânicos para manutenção da existência representa uma alternativa no direcionamento da obtenção de compostos 

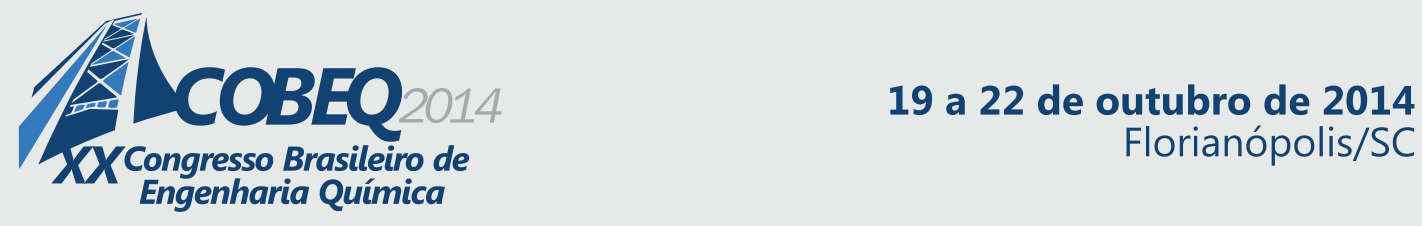

não obtidos em cultivos convencionais fotossintéticos, além de vantagens como a simplificação de operações unitárias do processo. Esse tipo de produção é suportada por carboidratos como glicose, frutose e sacarose (Perez-Garcia et al., 2011; Minerdi et al., 2009). As características qualitativas e quantitativas dos compostos orgânicos voláteis em microalgas são altamente influenciadas pelos parâmetros do sistema de cultivo.

É conhecido a partir de estudos ecofisiológicos que as microalgas produzem uma grande variedade de COVs que podem influenciar o aroma da biomassa (Draaisma et al., 2013). A técnica de extração por SPME é considerada eficaz e têm sido amplamente aplicada para a extração de compostos orgânicos voláteis e semivoláteis de amostras biológicas e produtos alimentares (Durme et al., 2013; Zhang et al., 2009). A caracterização da fração volátil dos biorreatores pode contribuir para o estabelecimento das rotas de bioconversão dos substratos, além de possibilitar a identificação de aplicações potenciais dos bioprodutos formados (Jacob-Lopes et al., 2010). 0 trabalho teve por objetivo avaliar a produção de COV's pela microalga Phormidium sp. em cultivo heterotrófico empregando glicose como fonte de carbono.

\section{MATERIAIS E MÉTODOS}

\subsection{Micro-organismo, meio de cultura, manutenção e propagação do inóculo.}

A cianobactéria utilizada foi a Phormidium sp., isolada do Deserto Cuatro Sienegas no México $\left(26^{\circ} 59^{\prime} \mathrm{N} 102^{\circ} 03 \mathrm{~W}\right)$. As culturas reservas foram mantidas e propagadas em agar-agar solidificado (20g. $\left.\mathrm{L}^{-1}\right)$ com meio sintético BG11 (RIPPKA et al, 1979) que possui a composição: K2HPO4 (0.03g. $\left.\mathrm{L}^{-1}\right), \mathrm{MgSO} 4$ (0.075g. $\left.\mathrm{L}^{-1}\right), \mathrm{CaCl} 2.2 \mathrm{H} 2 \mathrm{O}\left(0.036 \mathrm{~g} . \mathrm{L}^{-1}\right)$, citrato de amônio e ferro $\left(0.0006 \mathrm{~g} . \mathrm{L}^{-1}\right)$, Na2EDTA (0.001 g. $\left.\mathrm{L}^{-1}\right), \mathrm{NaCl}\left(0.00072 \mathrm{~g} . \mathrm{L}^{-1}\right)$, NaNO3 $\left(0.015 \mathrm{~g} . \mathrm{L}^{-1}\right)$, ácido cítrico $\left(0.0006 \mathrm{gL}^{-1}\right)$, $\mathrm{Na} 2 \mathrm{CO} 3$ (1.5g.L $\left.\mathrm{L}^{-1}\right)$, metais traços [H3BO3 (0.0028g.L $\left.{ }^{-1}\right), \mathrm{MnCl} 2.4 \mathrm{H} 2 \mathrm{O}\left(0.0018 \mathrm{~g} . \mathrm{L}^{-1}\right), \mathrm{ZnSO} 4.7 \mathrm{H} 2 \mathrm{O}$ (0.00022g.L $\left.\mathrm{L}^{-1}\right)$, Na2MoO4.2H2O (0.00039g.L $\left.\mathrm{L}^{-1}\right)$, CoSO4.6H2O (0.00004g.L $\left.\left.\mathrm{L}^{-1}\right)\right]$. As condições de manutenção usadas foram $25^{\circ} \mathrm{C}$ e intensidade luminosa constante de $1 \mathrm{klux}$.

\subsection{Cultivo Heterotrófico Microalgal}

Os experimentos foram realizados em um reator de coluna de bolhas. O sistema foi construído de vidro de borosilicato com diâmetro externo de 12.5 centímetros e altura de 16 centímetros, com razão altura/diâmetro proporção igual a 1.28 e um volume nominal de $2.0 \mathrm{~L}$. O sistema de dispersão do reator consistiu em um difusor de ar com 2.5 centímetros de diâmetro localizado no interior do bioreator. O fluxo de ar controlado pelo medidor de fluxo (Kl-Key Instruments®, Trevose, PA, EUA) e a entrada de ar e a saída de gases serão filtrados com unidades filtrantes constituídas de membrana de polipropileno, com um diâmetro de poro de $0.22 \mu \mathrm{m}$ e o diâmetro total de de $50 \mathrm{~mm}$ (Millex FG ${ }^{\circledR}$, Billerica, MA, EUA).

$\mathrm{O}$ biorreator, incluindo unidades filtrantes foram esterilizados em autoclave a $121^{\circ} \mathrm{C}$ durante 40 minutos. Os experimentos foram realizados em biorreatores em regime de batelada, alimentado com 2.0L de meio de cultura. As condições experimentais foram as seguintes: concentração inicial do inoculo de $100 \mathrm{mg} / \mathrm{L}$, a temperatura de $26^{\circ} \mathrm{C}$, o pH ajustado para 7.6, aeração de $0,1 \mathrm{VVM}$ (volume de 


\section{9 a 22 de outubro de 2014 \\ Florianópolis/SC}

ar por volume de cultura por minuto) em ausência de luz. A concentração de glicose foi ajustada a concentração de $12,5 \mathrm{gL}^{-1}$.

\subsection{Determinação de Compostos Orgânicos Voláteis}

Os compostos voláteis formados no bioprocesso foram isolados pela técnica de microextração em fase sólida aplicada em headspace (HS-SPME). A amostragem realizada no tempo zero e a cada 24h durante o crescimento celular. A fibra de SPME de revestimento misto empregada foi a DVB/Car/PDMS (divinilbenzeno/Carboxen/polidimetilsiloxano; 50/30 $\mathrm{m} \times 20 \mathrm{~mm}$, Supelco Bellefonte, PA, USA), pré-condicionada conforme as recomendações fornecidas pelo fabricante. A temperatura de extração de $40^{\circ} \mathrm{C}$, com um tempo de equilíbrio de 5 minutos, após exposição da fibra por 45 minutos. A análise dos compostos voláteis foi realizada em um cromatógrafo a gás acoplado a um espectrômetro de massas (GC/MS Shimadzu QP-2010 Plus). As separações cromatográficas em coluna Chrompack WAX 52-CB $(60 \mathrm{~m} \times 0,25 \mathrm{~mm}$ d.i. $\times 0,25 \mu \mathrm{m}$ de diâmetro de fase estacionária $)$. O

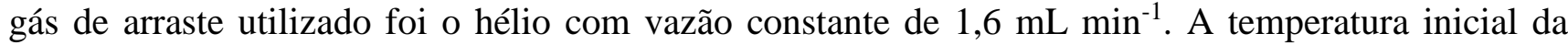
coluna de $35^{\circ} \mathrm{C}$, permanecendo por 5 minutos, após elevada até $220^{\circ} \mathrm{C}$ com gradiente de temperatura de $5^{\circ} \mathrm{C} / \mathrm{min}$, mantendo-se isotermicamente por 5 minutos. A interface $\mathrm{GC} / \mathrm{MS}$ e da fonte de ionização foram mantidos a $250^{\circ} \mathrm{C}$. $\mathrm{O}$ detector de massas foi operado no modo de ionização por elétrons, com feixe de elétrons a $+70 \mathrm{eV}$. $\mathrm{O}$ analisador de massas do tipo quadrupolos foi utilizado no modo de varredura na faixa de 35 a $350 \mathrm{~m} / \mathrm{z}$. Os compostos foram identificados primeiramente por comparação dos seus espectros de massa com os do banco de dados espectral da própria biblioteca do GC-MS (NIST MS Search 2.0). A identificação foi confirmada por comparação dos Índices de Retenção Linear calculados.

\section{RESULTADOS E DISCUSSÃO}

Os COVs identificados estão expressos na Tabela 1, juntamente com seus índices de retenção e porcentagem da área relativa dos picos. Foram identificados, na fase gasosa dos biorreatores heterotróficos microalgais, um total de 54 compostos, distribuídos entre aldeídos, álcoois, cetonas, éteres, ésteres e hidrocarbonetos. O tempo de residência de $96 \mathrm{~h}$ corresponde ao período das maiores taxas de formação destas biomoléculas.

Tabela 1 - Compostos orgânicos voláteis (COVs) detectados por GC/MS, índices de retenção linear (IR) e área relativa dos picos (\%).

\begin{tabular}{llllll}
\hline & & & \multicolumn{3}{c}{ Área Relativa $(\%)$} \\
\cline { 5 - 6 } Pico & Composto & IR & $0 \mathrm{~h}$ & $96 \mathrm{~h}$ & $144 \mathrm{~h}$ \\
\hline $\mathbf{0 1}$ & Éter Dietílico & 612 & 36,0 & 6,4 & 1,6 \\
\hline $\mathbf{0 2}$ & Éter metil-terc-butílico & 635 & 6,7 & 2,4 & 0,9 \\
\hline $\mathbf{0 3}$ & Acetoaldeído & 648 & $*_{\text {nd }}$ & 1,4 & 1,3 \\
\hline $\mathbf{0 4}$ & Octano & 806 & 0,8 & 0,1 & 0,1 \\
\hline $\mathbf{0 5}$ & Isobutanal & 826 & $*_{\text {nd }}$ & 0,1 & 0,1 \\
\hline $\mathbf{0 6}$ & Butanal & 895 & $*_{\text {nd }}$ & 0,1 & 0,1 \\
\hline
\end{tabular}




\begin{tabular}{|c|c|c|c|c|c|}
\hline 07 & Acetato de Etila & 905 & 2,4 & 1,3 & 1,3 \\
\hline 08 & 2-Butanona & 921 & 1,3 & 1,1 & 1,2 \\
\hline 09 & 3-Metil-2-butanona & 933 & $*$ nd & 0,1 & 0,1 \\
\hline 10 & Álcool Isopropílico & 948 & $*$ nd & 2,1 & 2,8 \\
\hline 11 & 2-Pentanona & 996 & 2,4 & 4,9 & 7,5 \\
\hline 12 & 2,3-Butanodiona & 1002 & 1,3 & 1,9 & 1,9 \\
\hline 13 & 4-Metil-2-pentanona & 1025 & $*$ nd & 0,2 & 0,1 \\
\hline 14 & Acetonitrila & 1027 & $*$ nd & 0,9 & 1,2 \\
\hline 15 & Trimetilsilanol & 1039 & 1,8 & 0,1 & 0,1 \\
\hline 16 & Isobutanol & 1115 & 1,9 & 2,8 & 3,6 \\
\hline 17 & Acetato de Isoamila & 1134 & $*$ nd & 0,1 & 0,1 \\
\hline 18 & 2,3-Hexanodiona & 1149 & $*$ nd & 0,1 & $*$ nd \\
\hline 19 & 2-Pentanol & 1151 & $*$ nd & 0,4 & 1,7 \\
\hline 20 & 2-Heptanona & 1193 & $*_{\text {nd }}$ & 0,1 & 0,1 \\
\hline 21 & Álcool Isoamílico & 1228 & 2,1 & 0,6 & $*$ nd \\
\hline 22 & 3-Octanona & 1263 & 2,0 & 0,3 & 4,2 \\
\hline 23 & 3-metil Butenol & 1269 & $*$ nd & 2,1 & 1,8 \\
\hline 24 & 3-Hidroxi 2-butanona & 1411 & $*$ nd & 12,0 & 7,0 \\
\hline 25 & 1-Hidróxi 2-Propanona & 1435 & $*$ nd & 0,4 & $*$ nd \\
\hline 26 & 6-metil 5-Hepten-2-ona & 1451 & 0,9 & 0,4 & 1,2 \\
\hline 27 & Hexanol & 1466 & 1,8 & 0,5 & 0,9 \\
\hline 28 & Metil 2-Hidroxi isovalerato & 1516 & $*$ nd & 1,3 & 1,1 \\
\hline 29 & 2-Butoxietanol & 1520 & 0,8 & 0,4 & 0,4 \\
\hline 30 & 2-Metil-3(2H)Furanona & 1536 & $*$ nd & 19,0 & 16,0 \\
\hline 31 & Heptanol & 1569 & 1,1 & 0,8 & 0,7 \\
\hline 32 & Ácido Acético & 1572 & $*$ nd & 9,9 & 7,8 \\
\hline 33 & 2 Etil Hexanol & 1601 & 5,6 & 2,4 & 1,8 \\
\hline 34 & Decanal & 1616 & 3,4 & 2,7 & 2,0 \\
\hline 35 & Etil 3-hidroxibutirato & 1645 & $*$ nd & 0,6 & 1,3 \\
\hline 36 & Mercaptoetanol 2-etil & 1662 & $*$ nd & 1,1 & 1,5 \\
\hline 37 & Octanol & 1675 & 1,9 & 0,8 & 0,6 \\
\hline 38 & Ácido Propanóico 2-metil & 1686 & $*$ nd & 1,8 & 2,8 \\
\hline 39 & Ácido Butanóico & 1751 & $*$ nd & 4,9 & 10,0 \\
\hline 40 & D-Neoisomentol & 1762 & 1,6 & 0,8 & 0,2 \\
\hline 41 & Nonanol & 1779 & 2,9 & 1,6 & 0,9 \\
\hline 42 & Ácido Isovalérico & 1790 & $*$ nd & 2,4 & 6,1 \\
\hline 43 & Acetofenona & 1796 & 9,0 & 1,3 & 1,2 \\
\hline 44 & Heptadecano & 1810 & 4,2 & 0,6 & 0,8 \\
\hline 45 & Decanol & 1882 & 1,0 & $*$ nd & $*$ nd \\
\hline 46 & 2-Fenilisopropanol & 1890 & 1,8 & 0,8 & 0,9 \\
\hline
\end{tabular}




\begin{tabular}{llllll}
\hline $\mathbf{4 7}$ & Formamida,N,N,dibutil & 1918 & 0,9 & 0,2 & 0,4 \\
\hline $\mathbf{4 8}$ & Acetato de Fenetila & 1945 & $*$ nd & 0,9 & 0,9 \\
\hline $\mathbf{4 9}$ & Álcool Benzílico & 2005 & 0,8 & 0,3 & 0,1 \\
\hline $\mathbf{5 0}$ & Dodecanol & 2059 & 1,4 & 0,6 & 0,6 \\
\hline $\mathbf{5 1}$ & Benzotiazol & 2081 & 0,6 & 0,2 & 0,1 \\
\hline $\mathbf{5 2}$ & 3-Buten-2-ona 4-(2,2,6-trimetil) & 2096 & 0,8 & 0,1 & $*$ nd \\
\hline $\mathbf{5 3}$ & Álcool Fenetílico & 2102 & 0,9 & 0,3 & 0,4 \\
\hline $\mathbf{5 4}$ & Ácido Hexanodióico & 2330 & $*$ nd & 1,4 & 0,7 \\
\hline
\end{tabular}

*não detectado.

A ocorrência da maior parte dos produtos voláteis pode ser atribuída a composição da biomassa microalgal. Do total de compostos identificados, as principais classes de compostos foram álcoois $(37,04 \%)$, cetonas $(25,92 \%)$, ácidos $(9,26 \%)$ e aldeídos $(7,41 \%)$. Inicialmente, foram detectados 30 compostos, sendo o éter dietílico, éter metil-terc-butílico e acetofenona os compostos de maior área respectivamente. Em 144 horas, ao fim do experimento, foram encontrados 49 compostos, desses os majoritários foram 2-metil-3(2H) furanona, os ácidos butanoico e acético.

Durante as análises, vinte e quatro compostos foram formados ao longo de $144 \mathrm{~h}$, destacaramse as cetonas $(29,17 \%)$, ácidos $(20,83 \%)$ e álcoois $(16,67 \%)$. Cinco compostos desapareceram, sendo esses: 1-decanol, álcool isoamílico,2,3-hexanodiona, 1-hidroxi-2-propanona e 2-buten-2-ona-4(2,2,6trimetil). Durme et al. (2013) avaliaram a composição volátil de microalgas e como compostos predominantes encontraram aldeídos, cetonas e álcoois, mostrando-se assim similar aos resultados obtidos dos biorreatores heterotróficos de Phormidium sp.. A caracterização volátil de biorreatores microalgais é de grande importância não somente para a elucidação das rotas metabólicas, bem como para a obtenção de biomoléculas que podem ter amplo uso em diversos segmentos industriais.

\section{CONCLUSÕES}

Com os resultados obtidos foi possível observar elevadas taxas de produção de álcoois, cetonas, ácidos e aldeídos em cultivos heterotróficos microalgais. A identificação destes compostos contribui para o conhecimento do metabolismo microalgal, abrindo possibilidades de exploração comercial destes metabólitos secundários.

\section{REFERÊNCIAS}

BOROWITZKA, M. A. High-value products from microalgae their development and commercialisation. Journal of Applied Phycology, v. 25, p. 743-756, 2013.

BURJA, A. M; BANAIGS, E. B.; MANSOUR, A.; BURGESS, J. G.; WRIGHT, P. C. Marine Microalgae: a prophilic source of natural products. Tetrahedron, v. 57, p. 9347-9377, 2001.

CHENG, Y.; ZHOU, W.; GAO, C.; LAN, K.; GAO, Y.; WU, Q. Biodiesel production from Jerusalem artichoke (Helianthus tuberosus L.) tuber by heterotrophic microalgae Chlorella protothecoides. Journal of Chemistry and Technology Biotechnology, v. 84, p. 777-781, 2009. 
DRAAISMA, R. B.; WIJFFELS, R. H.; SLEGERS, P. M.; BRENTNER, L. B., ROY, A.; BARBOSA, M. S. Food commodities from microalgae. Current Opinion in Biotechnology, v. 24, p. 169-177, 2013.

DURME, J. V.; GOIRIS, K.; DE WINNE, A.; DE COOMAN, L.; MEYLAERT, K. Evaluation of the volatile composition and sensory properties of five species of microalgae. Journal of Agriculture and Food Chemistry, v. 61, p. 10881-10890, 2013.

GUIRY, M. D.; GUIRY, G.M. AlgaeBase. World-wide eletronic publication, National University of Ireland, Galway, 2013. http://www.algaebase.org; pequisa realizada em 09 de dezembro de 2013.

JACOB-LOPES, E.; SCOPARO, C. H. G.; QUEIROZ, M. I.; FRANCO, T. T. Biotransformations of carbon dioxide in photobiorreactors. Energy Conversion and Management, v. 51, p. 894-900, 2010.

MINERDI, D.; BOSSI, S.; GULLINO, M. L.; GARIBALDI, A. Volatile organic compounds; a potential direct long distance mechanism for antagonistic action of Fusarium oxysporum strain MAS 35. Environmental Microbiology, v. 11, p. 844-854, 2009.

MORALES-SÁNCHEZ, D.; TINOCO-VALENCIA, R.; MARTINEZ, A. Heterotrophic growth of Neochloris oleoabundandans using glucose as a carbon source. Biotechnology for Biofuels, v. 6, p. 112, 2013.

PEREZ-GARCIA, O.; ESCALANTE, F. M. E.; DE-BASHAN, L. E.; BASHAN, Y. Heterotrophic cultures of microalgae: Metabolism and potential products. Water Research, v. 45, p. 11-36, 2011.

THOMAZEU, S.; HOUDAN-FOURMONT, A.; COUTÉ, A.; DUVAL, C.; COULOUX, A.; ROSSEAU, F.; BERNARD, C. The contribuition of sub-Saharan African strains to the phylogeny of cianobacteria focusing on the Nostocaceae (Nostocales, cyanobacteria). Journal of Phycology, v.46, p. 564-579, 2010.

WEN, Z.Y.; CHEN, F. Heterotrophic production of eicosapentaenoic acid by microalgae. Biotechnology Advances, v. 21, p. 273-294, 2003.

ZHANG, Z.; LI, T.; WANG, D.; ZHANG, L.; CHENG, G. Study on the colatile profile characteristics of oyster Crassostrea gigas during storage by a combination sampling method coupled with GC/MS. Food Chemistry, v. 115, p. 1150-1157, 2009. 